\title{
Identification of a Novel Coxsackievirus A16 Strain Isolated from the Clinic HFMD Sample in Hubei Province in 2010
}

\author{
Tingting Xu, Weixia Wang, Songya Lyu* \\ State Key Laboratory of Virology, College of Life Sciences, Wuhan University, Wuhan, China \\ Email address: \\ xtt@whu.edu.cn (Tingting Xu), wangweixiawh@163.com (Weixia Wang),00006510@whu.edu.cn (Songya Lyu) \\ ${ }^{*}$ Corresponding author
}

To cite this article:

Tingting Xu, Weixia Wang, Songya Lyu. Identification of a Novel Coxsackievirus A16 Strain Isolated from the Clinic HFMD Sample in Hubei Province in 2010. Advances in Bioscience and Bioengineering. Vol. 4, No. 5, 2016, pp. 51-58. doi: 10.11648/j.abb.20160405.12

Received: August 25, 2016; Accepted: September 26, 2016; Published: October 15, 2016

\begin{abstract}
Enterovirus 71 (EV71) and Coxsackievirus A16 (CoxA16) are two major pathogens of Hand, foot and mouth disease (HFMD) that epidemic periodically among children and cause deaths occasionally. There is no effective vaccine or drug for HFMD to date. Here the whole genome of a clinic virus isolate GDV126 (China Center for Type Culture Collection, CCTCC) of HFMD was sequenced in Hubei 2010. Phylogenetic analysis demonstrates that the virus belongs to CoxA16. Both phylogenetic and recombination analysis of GDV126 showed that the GDV126 was a recombinant strain of CoxA16. In addition, GDV126 had different cell tropism from EV71. This work demonstrated the genetic variations and evolution of the HFMD pathogens and implicated complication to prevent the disease.
\end{abstract}

Keywords: Coxsackievirus A16, Hand, Foot and Mouth Diseases, Phylogenetic Analysis

\section{Introduction}

Hand, foot and mouth diseases (HFMD) are epidemic periodically among children under five years of age [1]. Children suffering from EV71 infection might develop severe complications such as acute flaccid paralysis, myocarditis or even fatal encephalitis. However, compared with EV71, CA16-associated HFMD is usually mild and benign $[2,3,4]$. HFMD was first reported in California in 1969 (Schmidt NJ et al., 1974) and then broke in Europe countries such as Bulgaria in 1975 and Hungarian in 1978 [5, 6]. Within last two decades, HFMD took large outbreaks many times in Asia countries including Malaysia in 1997, Taiwan in 1998, Japan in 2000, Vietnam in 2005 and mainland China in 2008. Therefore, HFMD has drawn considerable attention from the Ministry of Health of China. HFMD was classified as a category C notifiable infectious disease, and a virological surveillance system was set up in 2008 [7].

The Enterovirus genus comprises 12 species groups, which include 4 human enterovirus species (A-D) [8]. EV71 is a member of the enterovirus species A, along with CVA2-8, CVA10, CVA12, CVA14, and CA16. The single-stranded, positive-sense RNA genome is approximately $7.4 \mathrm{~kb}$ in size.
The $\mathrm{P} 1$ region encodes structural proteins (VP1, VP2, VP3, and VP4), while P2 and P3 regions encode non-structural proteins $2 \mathrm{~A}-2 \mathrm{C}$ and $3 \mathrm{~A}-3 \mathrm{D}$, respectively. Based on $\mathrm{VP} 1$ gene sequence, EV71 is further divided into groups $\mathrm{A}, \mathrm{B}$, and $\mathrm{C}$. Group A consists of the prototype $\mathrm{BrCr}$ strain, while groups $\mathrm{B}$ and $\mathrm{C}$ are subdivided into subgroups B1-B5 and $\mathrm{C} 1-\mathrm{C} 5$, respectively [9]. In 2014, the EV71 vaccines have achieved success in phase III trials $[10,11]$.

In this paper, the whole genome of a clinic virus strain of HFMD isolated Hubei province in 2010, named GDV126 (sample number of China Center for Type Culture Collection (CCTCC)) was sequenced. Both phylogenetic and recombination analysis of GDV126 showed that the GDV126 was a recombinant strain of CoxA16 (Figure 1,2). In addition, GDV126 had different cell tropism from EV71. This work may provide some clues of the genetic variations and evolution of the HFMD pathogens and implicate complication to prevent the disease.

\section{Materials and Methods}

GDV126 strain was got from China Center for Type Culture Collection (CCTCC). The virus was duplicated in African green monkey kidney (Vero, China) cells in DMEM (Gibco, 
USA) supplemented with 2\% FBS (Gibco, USA), 100U/ml penicillin and $10 \mu \mathrm{g} / \mathrm{ml}$ streptomycin (Introgen, USA) at MOI 0.2 . Infectious supernant was collected $72 \mathrm{hpi}$.

To further characterize this virus, the full-length viral genome of GDV126 extracted form the supernant using Trizol regent was determinated through reverse transcription (RT) PCR with eight sets of overlapped segment-specific primers that were designed according to the conserved nucleotides. First strand cDNA synthesis was undertaken using SuperScript II reversetranscriptase (Invitrogen, USA); viral cDNA was amplified using PCR SuperMix (Invitrogen, USA) with gene-specific primers and gel purified using a MinElute gel extraction kit (Qiagen, Germany). The terminal sequences were confirmed by rapid amplification of cDNA ends using a 5'/3' rapid amplification of cDNA ends kit (Roche, Mannheim, Germany).

To better understand viral evolutionary status, phylogenetic trees were established using the GDV126 full genome and some other full genome sequences of human enterovirus 71 and virus in GenBank through the MEGA5 and ClusterX software. The phylogenetic relationships were calculated by the neighbor-joining method. Nucleotide substitutions were set under the Kimura 2-parameter model. The nucleotide sequences used for the phylogenetic analysis were as follows: 5'-UTR, P1, P2 and $\mathrm{P} 3$ region.

To figure out the cell susceptibility of GDV126 virus, Vero, RD, U251, Mrc-5 and Thp-1 cell lines which are commonly used were choosen to isolate enterovirus for the infection test.

\section{Results and Discussions}

\subsection{Whole Genome Sequence of the Virus Isolate}

By gene sequencing, the whole genomic length of GDV126 was 7404 nucleotides. The complete genome sequences of GDV126 were deposited under GenBank (Accession NO. KU163608). Comparing our sequencing results to other enterovirus gemones in GenBank by Blast, we found that the nucleotide of GDV126 showed 92\% identity with another recombinant human Coxsackievirus A16 strain SZ/HK08-7 [12] (GenBank:GQ279371.1).

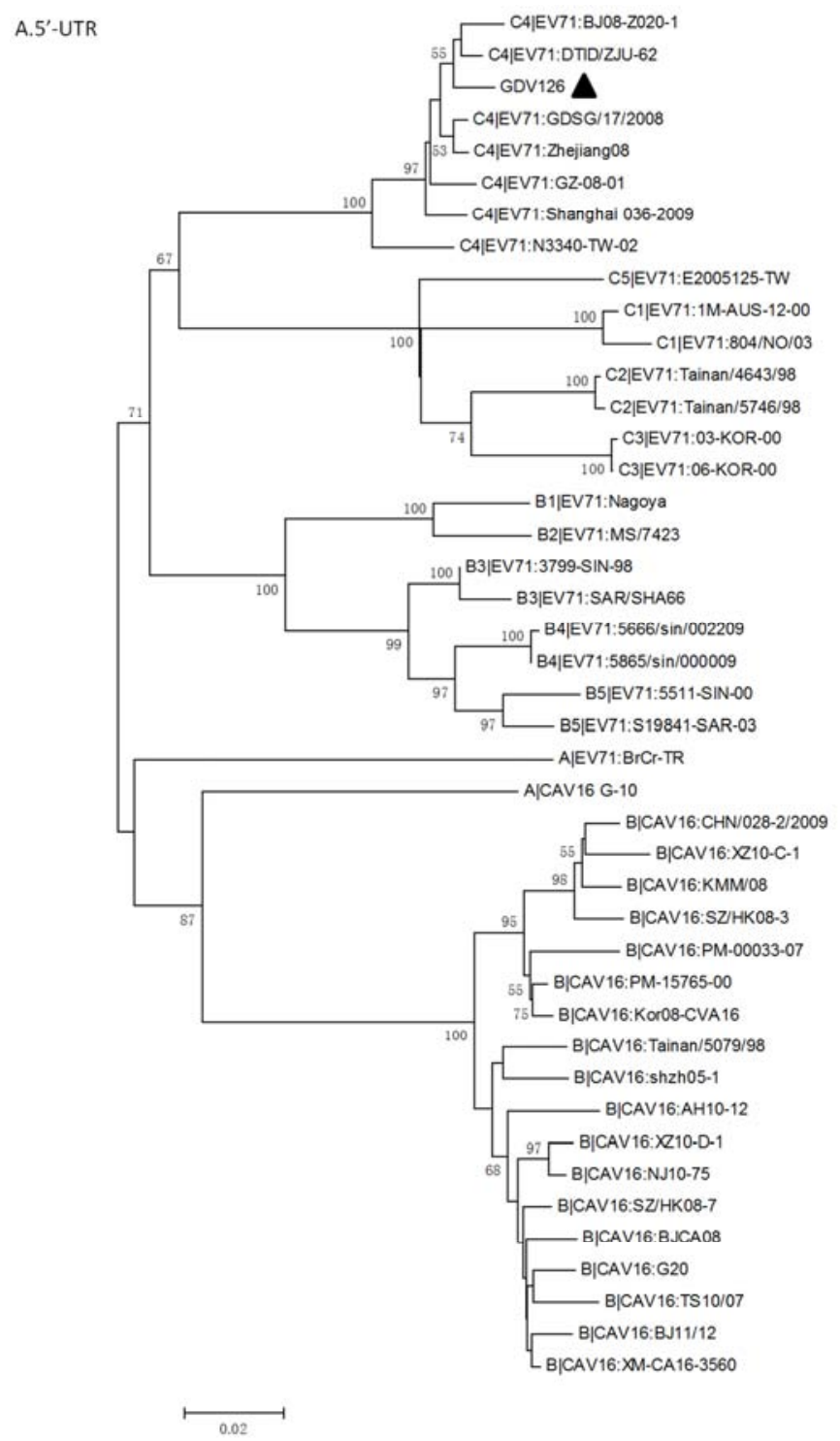


B.P1

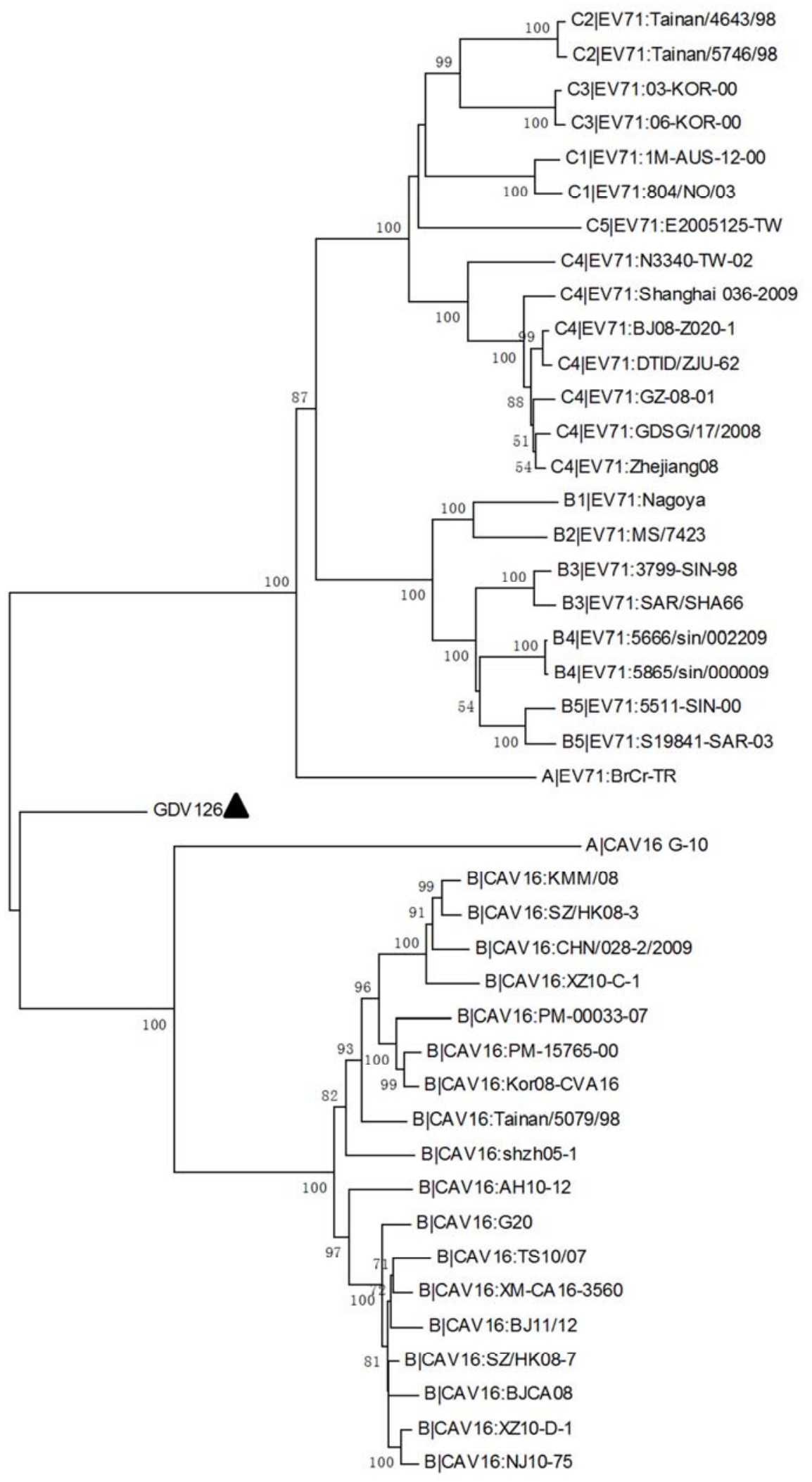


C.P2

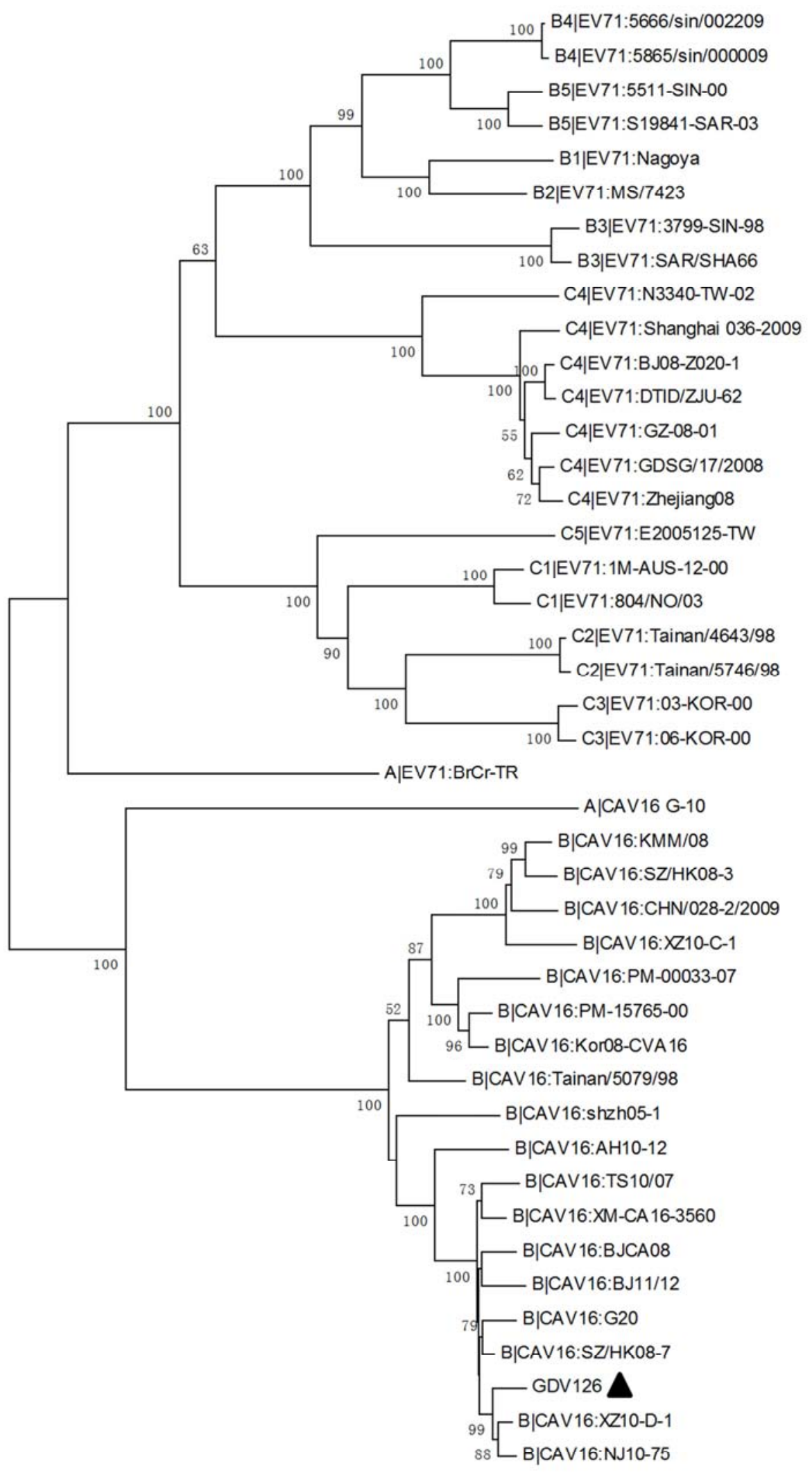


D.P3

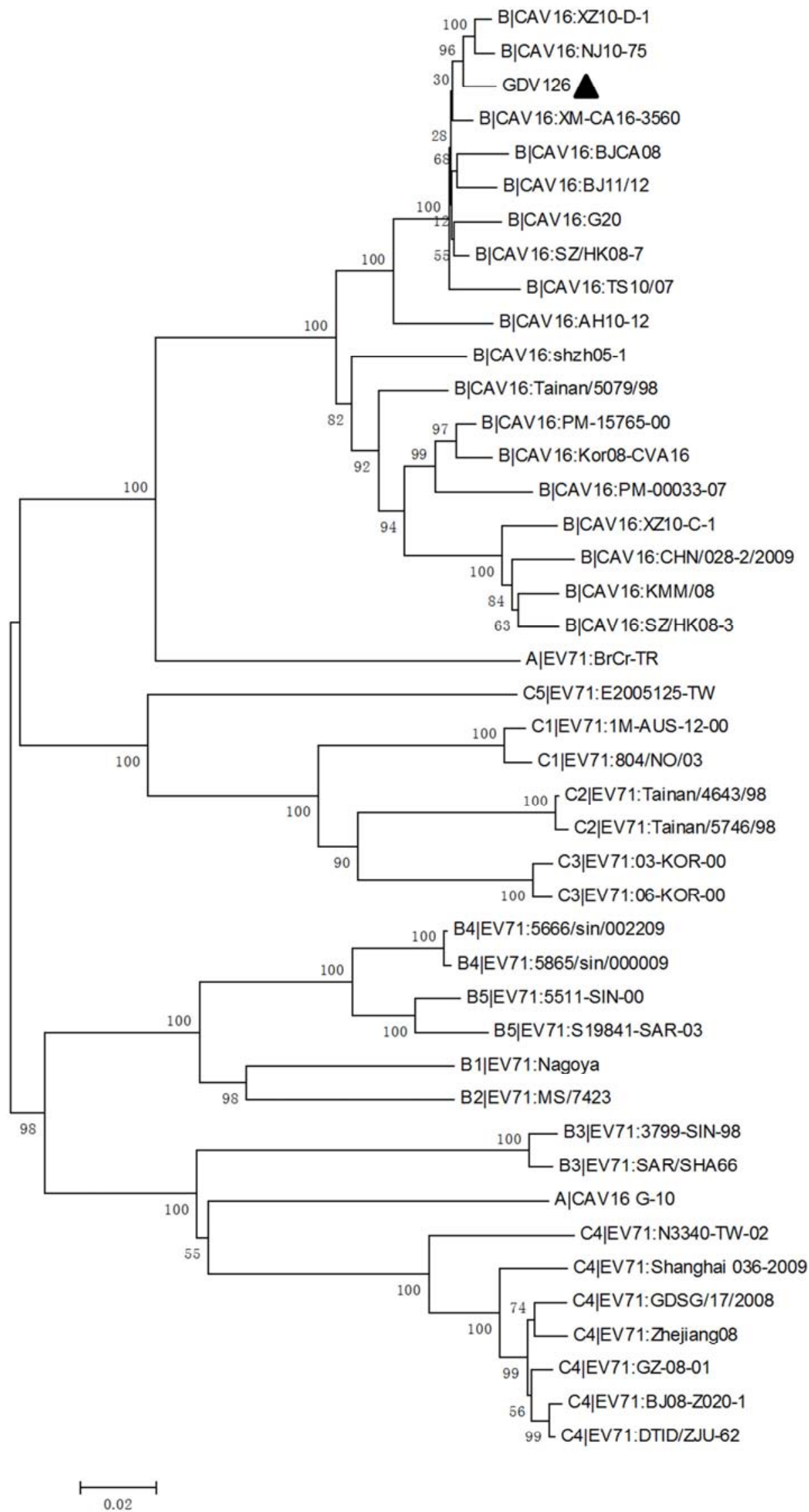

Figure 1. Phylogenetic analysis of the 5'-UTR, P1, P2 and P3 region of GDV126 virus with other EV71 and viruses in GenBank. The phylogenetic relationships were calculated by the neighbor-joining method. Bootstrap values expressed as percentage are shown at the nodes.Viral full names were shown in the tree and tested values $>50 \%$ were shown in the nodes. The GDV126 obtained in this study was labeled by triangle. 


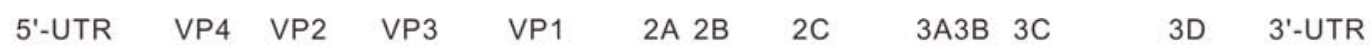
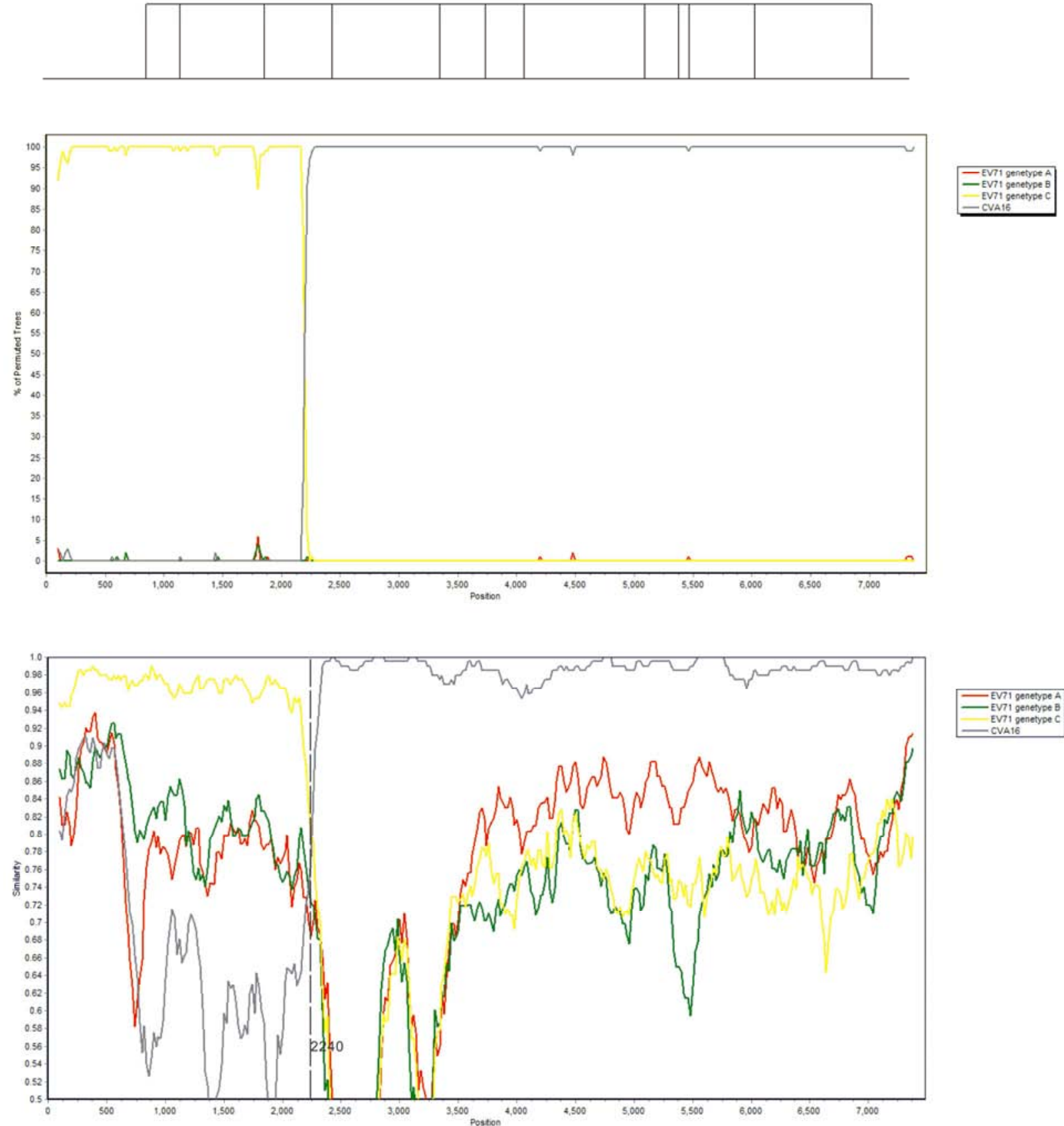

Figure 2. Recombination analysis of the GDV126 complete genomes. Bootscanning (upper panel) and similarity plot analysis (lower panel) were conducted with SimPlot version 3.5 .1 (Kimura distance model; window size, 1,000 bp; step, $20 \mathrm{bp}$ ), generated with MEGA version 5.1.

\subsection{Phylogenetic Analysis and Bootscan Results}

Phylogenetic analysis showed that GDV126 was clustered with EV71 genotype C4 strains for the 5'-UTR region, while the $\mathrm{P} 1$ region of GDV126 is independent of EV71 and CA16. Intriguingly, the P2 and P3 of GDV126 virus has a high bootstrap with Coxsackievirus A16 strain XZ10-D-1.

Since the incongruent phylogenetic relationships observed among different region of genome suggested that recombination events may have taken place in GDV126.Similarity plot and bootscan analyses were performed to identify potential recombination sites (Figure 2).
In the similarity plot analysis, GDV126 showed high sequence similarity ( $\geq 94 \%$ ) to EV71 genotype C before position 2240 . However, a higher similarity ( $\geq 96 \%$ ) to CAV16 after position 2240 . In the bootscan analysis, the results showed that from 5' end of the genome to position 2160, high bootstrap support for clustering between EV71 genotype C and GDV126. From position 2280 to the 3 ' end of the genome, high bootstrap support for clustering between CAV16 and GDV126 was observed. These findings indicated that recombination events has possibly taken place between nucleotide 2160 and 2280, corresponding to the VP3 region. 


\subsection{Different Cell Sensitivity of the Virus Isolates}

To figure out the cell susceptibility of GDV126 virus, we choose Vero, RD, U251, Mrc-5 and Thp-1 cell lines which are commonly used to isolate enterovirus for the infection test. The results was shown in Table $1(+$ : cell are sensitive to vires,-: cell are insensitive to virus). We found that RD was not sensitive to the GDV126 infection which is different from other study [13]. Interestingly, GDV126 could infect Thp-1 cells but EV71 could not. The results indicated that more than one cell lines should be used in order to isolate the virus strains from certain specimen of HFMD. In the future, more cell lines sensitive to human enterovirus infection will be tested to determine the cellular spectrum of GDV 126.

Table 1. Cell susceptibility of EV71 and GDV126 virus.

\begin{tabular}{llllll}
\hline & Vero & RD & U251 & Mrc-5 & Thp-1 \\
\hline EV71 & + & + & + & + & - \\
GDV126 & - & - & + & + & + \\
\hline
\end{tabular}

\section{Conclusions}

For enteroviruses, genetic recombination is a frequently phenomenon in picornaviruses which generates evolutionary diversity and also is a means to acquire new phenotypic traits which could result in the emergence of viruses with altered pathogenic potentials [14]. Phylogenetic analysis suggested that intertypic recombination event may have occurred between CA16 and EV71 genotype C, which was similar to other studies [15, 16, 17]. Meanwhile, similarity plot and bootscan analyses demonstrated that recombination events has possibly taken place between nucleotide 2160 and 2280, corresponding to the VP3 region. It is reported that recombination was frequently detected in the $\mathrm{P} 2$ and $\mathrm{P} 3$ regions while it was rare in $\mathrm{P} 1$ region, probably because of the structural proteins was important for the virus replication and assembling of proper viral capsid [18, 19, 20]. In addition, it was determined that neutralization epitopes resided mainly on capsid proteins, especially protein VP1 [21]. The cross-react neutralization epitopes mighty reside on the proteins VP2 and VP3 [22]. Thus, our study may contribute to designing serological diagnostic test and vaccine to the prevalent CA16 strains and provide some clues of the genetic variations and evolution of the HFMD pathogens and implicate complication to prevent the disease.

\section{Acknowledgments}

This research has been supported by the grant from National Natural Science Foundation of China (No.31070147). The authors declare that they have no conflict of interest.

\section{References}

[1] Ho M, Enterovirus 71: the virus, its infections and outbreaks. J Microbiol Immunol Infect, 2000. 33 (4): 205-16.
[2] Cardosa M J, Perera D, Brown B A et al., Molecular epidemiology of human enterovirus 71 strains and recent outbreaks in the Asia-Pacific region: comparative analysis of the VP1 and VP4 genes. Emerg Infect Dis, 2003.9 (4): 461-8.

[3] Chan L G, Parashar U D, Lye M S et al., Deaths of children during an outbreak of hand, foot, and mouth disease in sarawak, malaysia: clinical and pathological characteristics of the disease. For the Outbreak Study Group. Clin Infect Dis, 2000. 31 (3): 678-83.

[4] Perez-Velez C M, Anderson M S, Robinson C C et al., Outbreak of neurologic enterovirus type 71 disease: a diagnostic challenge. Clin Infect Dis, 2007. 45 (8): 950-7.

[5] Nagy G, Takatsy S, Kukan E et al., Virological diagnosis of enterovirus type 71 infections: experiences gained during an epidemic of acute CNS diseases in Hungary in 1978. Arch Virol, 1982. 71 (3): 217-27.

[6] Shindarov L M, Chumakov M P, Voroshilova M K et al., Epidemiological, clinical, and pathomorphological characteristics of epidemic poliomyelitis-like disease caused by enterovirus 71. J Hyg Epidemiol Microbiol Immunol, 1979. 23 (3): 284-95

[7] Solomon T, Lewthwaite P, Perera D et al., Virology, epidemiology, pathogenesis, and control of enterovirus 71 . Lancet Infect Dis, 2010. 10 (11): 778-90.

[8] Mauleekoonphairoj J, Vongpunsawad S, Puenpa J et al., Complete genome sequence analysis of enterovirus 71 isolated from children with hand, foot, and mouth disease in Thailand, 2012-2014. Virus Genes, 2015. 51 (2): 290-3.

[9] Liu Y, Zhang F, Fu C et al., Combination of intratypic and intertypic recombinant events in EV71: a novel evidence for the "triple-recombinant" strains of genotype A viruses in Mainland China from 2008 to 2010. Virus Genes, 2015. 50 (3): 365-74.

[10] Li R, Liu L, Mo Z et al., An inactivated enterovirus 71 vaccine in healthy children. N Engl J Med, 2014. 370 (9): 829-37.

[11] Zhu F, Xu W, Xia J et al., Efficacy, safety, and immunogenicity of an enterovirus 71 vaccine in China. N Engl J Med, 2014. 370 (9): 818-28.

[12] Yip C C, Lau S K, Zhou B et al., Emergence of enterovirus 71 "double-recombinant" strains belonging to a novel genotype D originating from southern China: first evidence for combination of intratypic and intertypic recombination events in EV71. Arch Virol, 2010. 155 (9): 1413-24.

[13] Yang T.Xie T H.Song et al., Sensitivities of five kinds of cells to coxsackievirus A16 and enterovirus 71. International Journal of Biologicals, 2015(4)

[14] Simmonds P and Welch J, Frequency and dynamics of recombination within different species of human enteroviruses. J Virol, 2006. 80 (1): 483-93.

[15] Mao Q, Wang Y, Yao X et al., Coxsackievirus A16: epidemiology, diagnosis, and vaccine. Hum Vaccin Immunother, 2014. 10 (2): 360-7.

[16] Sun T, Liu Y, Zhang Y et al., Molecular phylogeny of coxsackievirus A16. J Clin Microbiol, 2014. 52 (10): 3829-30.

[17] Zhao K, Han X, Wang G et al., Circulating coxsackievirus A16 identified as recombinant type A human enterovirus, China. Emerg Infect Dis, 2011.17 (8): 1537-40. 
[18] McWilliam Leitch E C, Cabrerizo M, Cardosa J et al., The association of recombination events in the founding and emergence of subgenogroup evolutionary lineages of human enterovirus 71. J Virol, 2012. 86 (5): 2676-85.

[19] Chen X, Zhang Q, Li J et al., Analysis of recombination and natural selection in human enterovirus 71. Virology, 2010. 398 (2): 251-61.

[20] Wang X, Zhu C, Bao W et al., Characterization of full-length enterovirus 71 strains from severe and mild disease patients in northeastern China. PLoS One, 2012. 7 (3): e32405.
[21] Liu Q, Ku Z, Cai Y et al., Detection, characterization and quantitation of coxsackievirus A16 using polyclonal antibodies against recombinant capsid subunit proteins. J Virol Methods, 2011. 173 (1): 115-20.

[22] Chen X, Tan X, Li J et al., Molecular epidemiology of coxsackievirus A16: intratype and prevalent intertype recombination identified. PLoS One, 2013. 8 (12): e82861. 